\title{
Software-Defined Cooking Using a Microwave Oven
}

By Haojian Jin, Jingxian Wang, Swarun Kumar, and Jason Hong

\begin{abstract}
Despite widespread popularity, today's microwave ovens are limited in their cooking capabilities, given that they heat food blindly, resulting in a nonuniform and unpredictable heating distribution. We present software-defined cooking (SDC), a low-cost closed-loop microwave oven system that aims to heat food in a software-defined thermal trajectory. SDC achieves this through a novel high-resolution heat sensing and actuation system that uses microwave-safe components to augment existing microwaves. SDC first senses the thermal gradient by using arrays of neon lamps that are charged by the electromagnetic (EM) field a microwave produces. SDC then modifies the EM-field strength to desired levels by accurately moving food on a programmable turntable toward sensed hot and cold spots. To create a more skewed arbitrary thermal pattern, SDC further introduces two types of programmable accessories: A microwave shield and a susceptor. We design and implement one experimental test bed by modifying a commercial off-the-shelf microwave oven. Our evaluation shows that SDC can programmatically create temperature deltas at a resolution of $21^{\circ} \mathrm{C}$ with a spatial resolution of $3 \mathrm{~cm}$ without the programmable accessories, and $183^{\circ} \mathrm{C}$ with them. We further demonstrate how an SDC-enabled microwave can be enlisted to perform unexpected cooking tasks: Cooking meat and fat in bacon discriminatively and heating milk uniformly.
\end{abstract}

\section{INTRODUCTION}

Since the introduction of microwaves to the consumer market in the 1970s, they have seen widespread adoption and are today the third most popular domestic food heating method (after baking and grilling). ${ }^{13}$ Indeed, the original patents for the microwave by Raytheon Inc. in the late 1940s envisioned a universal food cooking instrument for all kinds of food ranging from meat to fish. ${ }^{1}$ While microwaves have revolutionized the kitchen since their inception, today's consumer microwaves are mainly used as blunt heating appliances (e.g., reheating pizzas) rather than precise cooking instruments (e.g., cooking steak). The potential of microwave cooking is limited by the fact that today's microwaves heat food blindly, resulting in a nonuniform and unpredictable heating distribution. ${ }^{18}$

We present software-defined cooking (SDC), a novel lowcost closed-loop system that can augment existing consumer microwaves to sense and control heating at a fine-grained resolution, all using microwave-safe components within the chamber. SDC's design can unlock numerous programmable heating opportunities (see Figure 1). For example, when microwaving liquids (e.g., milk, baby formula), one need not worry about uneven heating that may scald the mouth or destroy nutrients-a reason why microwaves should not be used to heat formula despite their convenience. Further, SDC can enable fine-grained forms of cooking. For example, the Maillard reaction occurs when searing meats and panfrying vegetables are an important part of the flavor of these cooked foods. With SDC, it is possible to execute a computergenerated Maillard reaction pattern that discriminatively heats the food surface.

At the heart of SDC is a novel approach both to sense and control heat at different points in space within the microwave chamber. SDC senses heat using the same phenomenon that produces heat in the first place: The electromagnetic (EM) field. SDC aims to measure the amplitude of the EM field at any given point. Although battery-powered sensors exist to sense both heat and EM fields, SDC must do so using only microwave-safe components, which excludes typical commercial batteries. ${ }^{6}$

SDC achieves this by relying on the fact that the EM field within the microwave is a natural source of energy. This means that one can simply power the sensor of the EM field by the EM field itself. SDC uses tiny radio frequency (RF)-powered

Figure 1. Results of microwaving wet thermal paper (black spots indicate high heat). (Top) A traditional microwave without/with a turntable. A turntable can mitigate uneven heating, but cold/hot spots remain. (Bottom) SDC, for uniform heating (fewer black spots show heat is spread uniformly) or heating to write "MobiCom." We use patterned susceptors and let SDC ensure the text area has been heated in hot spots.
No Turntable

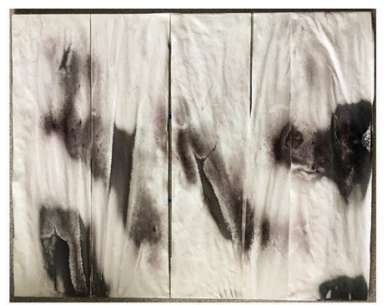

SDC Uniform Heating

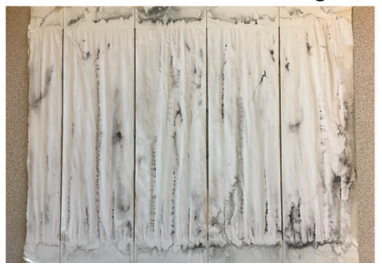

Default Turntable

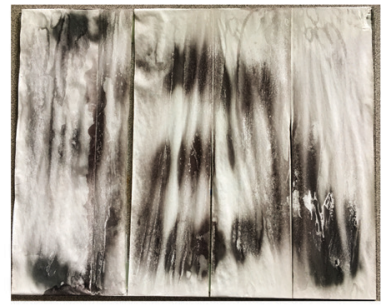

SDC Arbitrary Heating

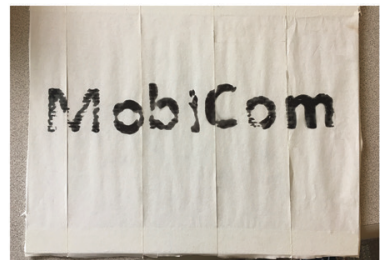

The original version of this paper was published in Proceedings of the $25^{\text {th }}$ Annual International Conference on Mobile Computing and Networking, 2019, ACM. 
neon lights (see Figure 2) that glow in response to the EM field within the microwave. Specifically, the oscillating microwave results in a potential difference (of a few $100 \mathrm{~V}$ to a few $\mathrm{kV}$ ) between two electrodes within each light bulb. Due to the potential difference, electrons are accelerated away from the cathode and give rise to collisions with the neon gas atoms or molecules, which will emit a characteristic glow in proportion to the amplitude of the field. Neon lights are inexpensive, compact, and produce minimal disruption to the EM field itselfmeaning that they can be tightly packed at key locations around the chamber to sense the EM-field amplitude at high accuracy. Given that the neon lights may be obstructed from view due to the food placed in the microwave, we run optical fibers made of microwave-safe glass that carry the light signals outside the chamber to be sensed by a camera (see Figure 3 ). Section 4 describes our framework to fuse measurements from this hardware with infrared (IR) cameras to estimate current and future spatial temperature distributions of the food.

\section{Figure 2. We place neon lights with different lengths of wire extensions (red: $0 \mathrm{~mm}$, green: $7.5 \mathrm{~mm}$, and blue: $15 \mathrm{~mm}$ ) under the turntable. We then measure the percentage of glowing time to quantify the sensitivity.}

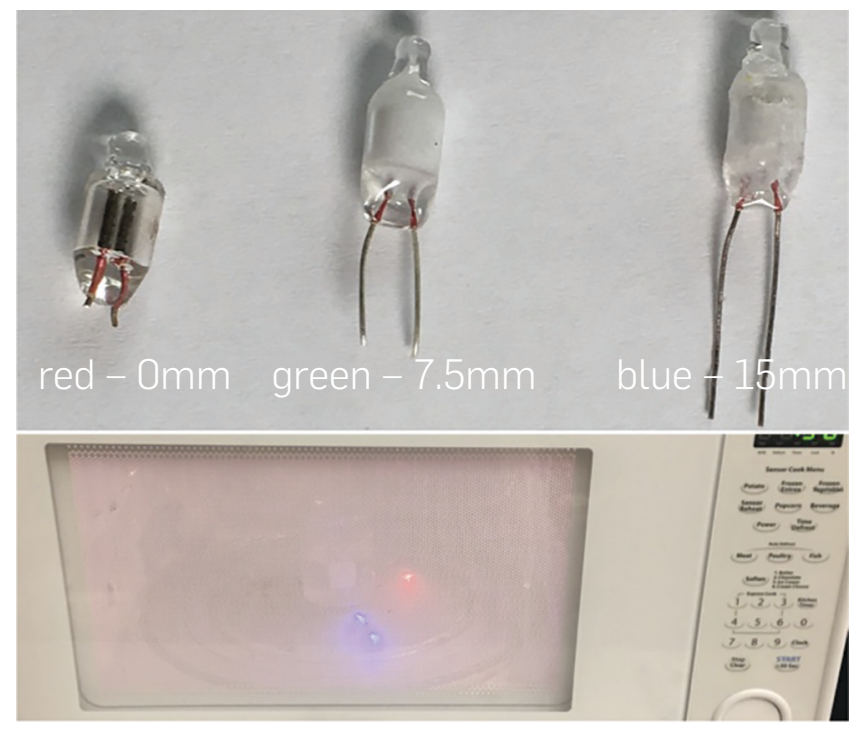

Figure 3. SDC's Hardware.

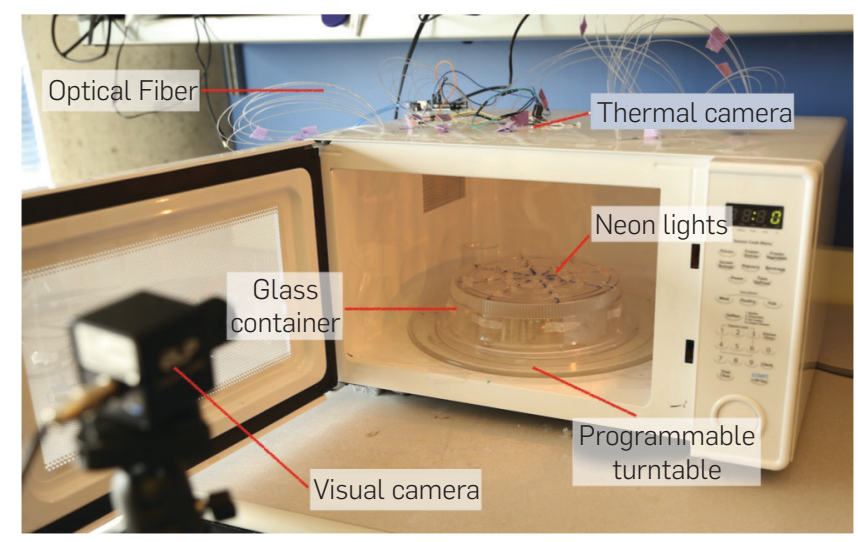

Upon sensing food heating, SDC controls heat according to the user-specified thermal recipe by building microwave shields that protect regions of the food that must not be overcooked (e.g., for meat). SDC achieves this through small metallic spheres placed within the microwave at key locations. Although conventional wisdom says that one must not place metal in a microwave, $\mathrm{RF}$ propagation is more nuanced. Specifically, metallic surfaces within the microwave only produce energetic sparks at sharp edges, found in most kitchen utensils and bowls. Metallic spheres by definition do not have edges and are thus microwave-safe. ${ }^{16}$ SDC carefully packs metallic spheres at specific regions of the microwave to minimize RF energy transfer at these regions. Section 5 shows how SDC accurately rotates the turntable to guide parts of the food that must not be overcooked toward these regions.

We implement a prototype of SDC by modifying a commercial Microwave oven (Sharp SMC1441CW). We place neon lamp arrays inside the microwave oven cavity and use a camera outside the cavity to monitor lamp flashes conducted via fiber-optic cables. We replace the coarse turntable motor with a step motor controllable via an Arduino board. Given a desired heating distribution pattern, SDC recommends the initial position where the user should place the food. During the heating, SDC continuously senses the real-time EM-field strength around the food and adjusts the actuation plan. Figure 3 illustrates the basic hardware setup of SDC. We conducted detailed experiments to evaluate SDC's sensing, uniform heating, and planned heating capabilities. Our experiments reveal:

- SDC can improve thermal heating uniformity by $633 \%$ compared to commercial microwaves with turntables.

- SDC can create an arbitrary temperature delta of $183^{\circ} \mathrm{C}$ with a resolution of $3 \mathrm{~cm}$.

- We demonstrate SDC in performing two unconventional cooking tasks: Cooking bacon and warming milk for an infant.

Contributions: SDC is a novel redesign of the microwave oven that both senses and actuates the EM field at fine-grained spatial resolution. SDC introduces programmable RF-powered neon lights whose signals are conducted by optic fibers that are microwave-safe to sense the EM-field distribution. SDC then adjusts the spatial heat distribution within the microwave chamber by moving food carefully around hot and cold spots predesigned using microwave-safe accessories. We built a prototype implementation of SDC by modifying an existing commercial microwave, and found that it had an accuracy of $7^{\circ} \mathrm{C}-10^{\circ} \mathrm{C}$ with respect to a heating recipe.

\section{MICROWAVE HEATING 101}

In a microwave, water, fat, and other electric dipoles in the food will absorb energy from the microwaves in a process called dielectric heating. ${ }^{21}$ Namely, when an electric field is applied, the bipolar molecules tend to behave like microscopic magnets and attempt to align themselves with the field. When the electrical field changes millions of times per second (e.g., 2450 million times per second for $2.45 \mathrm{GHz}$ microwave signals), these molecular magnets are unable to 
keep up in the presence of forces acting to slow them. This resistance to the rapid movement of the bipolar molecules creates friction and results in heat dissipation in the material exposed to the microwave radiation.

Although strong direct microwave radiation can burn human body tissue as well as electronic devices, the cooking chamber works as a Faraday cage to significantly attenuate waves escaping the microwave chamber. The US federal emission standard ${ }^{17}$ limits the amount of microwave leakage from an oven throughout its lifetime to $5 \mathrm{~mW}$ per square centimeter at approximately 2 inches from the oven surface (a safety factor of 10,000 or more below levels that may harm people ${ }^{10}$ ).

Once the microwave signals enter the metal cavity, they are effectively reflected by the metallic walls. Original and reflected waves resonate in the cavity and form standing waves ${ }^{19}$ which produce antinodes (heating hot spots) and nodes (heating cold spots). The EM fields are weak at nodes and therefore nothing cooks there. In contrast, EM fields at antinodes alternate at maximum amplitude to produce maximum heating. This is also the reason why microwaves have a rotating turntable so that the turntable moves food in and out of the hot spots to cook more uniformly.

\section{SDC HEATING RECIPE}

At its most basic, "cooking" means applying heat to food, ${ }^{12}$ which can be specified in three main variables:

- The most important variable in cooking is the temperature of food, which will trigger different chemical reactions (e.g., protein denaturation, Maillard reaction, and caramelization). For instance, if we want to cook a steak at least rare, we need to heat the meat to a temperature between $55^{\circ} \mathrm{C}$ (the highest survival temperature for most bacteria) and $65.5^{\circ} \mathrm{C}$ (the denaturing temperature for the protein actin).

- Time is an important factor in both cooking food accurately and killing bacteria. For example, the standard food safety rule ${ }^{4}$ provided by the Food and Drug Administration (FDA) states various time and temperature combinations: Heating at $55^{\circ} \mathrm{C}$ for $89 \mathrm{~min}$ can achieve a similar effect as heating at $62.2^{\circ} \mathrm{C}$ for $5 \mathrm{~min}$ to reduce Salmonella.

- Space: Different parts of food (e.g., meat vs. fat, egg white vs. yolk) may need to be cooked with different specifications to obtain optimal tasting food. SDC therefore aims to specify heating requirements for each spatial "pixel" of the food surface.

We envision that the future microwave heating recipes specify the desired thermal trajectory, that is, temperature vs. time, for each "pixel" of the food (see Figure 4 for an example steak recipe). This is precisely SDC's input, with its performance dictated by how closely it follows this specification.

We note that across all three common heating methods (convection, conduction, and radiation), food is cooked from the outside in, that is, the outer portions will warm up faster, and the heat conduction will heat the inner parts over time. So SDC focuses on controlling the surface temperature of food.

\section{SDC'S HEAT SENSING}

SDC's heat sensing aims to capture both the current temperature of food as well as the intent to heat. At first blush, one may assume that heat sensing can be readily achieved using a thermal camera that captures the current temperature of the food. By measuring thermal camera readings over time, one can make predictions about how food will heat in the future. Yet, thermal cameras have important limitations that limit a design that relies exclusively on them for heat sensing. First, the food thermal properties evolve on a time scale of seconds, and the carryover in cooking will continue heating even if the food is removed from the source of heat. However, thermal cameras often have limited refresh rates $(<9 \mathrm{~Hz})$ and modest accuracy $\left( \pm 2^{\circ} \mathrm{C}\right)$. Therefore, thermal cameras only measure the effect of heating after-the-fact and cannot prevent undesired heating, often until the damage is already done. Thermal cameras are also limited to measuring heating on the surface of the food in direct line-of-sight. To mitigate this, SDC complements a thermal camera that senses current temperature with microwave-safe sensors that estimate future expected temperature (see Figure 5).

\subsection{Sensing hardware design}

SDC places an array of neon lights, each with a 5-mm diameter and 13-mm length, inside the microwave chamber to sense EM fields. A neon light (see Figure 2) is a miniature gas discharge lamp, which consists of a small glass capsule that contains a mixture of neon and other gases at low pressure as well as two electrodes (an anode and a cathode). During microwaving, the electrodes will couple with the EM field and act as antennas. The oscillating microwave applies a potential difference between two electrodes. Due to the potential

Figure 4. SDC heating recipe is a progression of desired temperature vs. time per pixel of food. For example, if we want to cook a steak at least rare, the ideal temperature is between $55^{\circ} \mathrm{C}$ and $65.5^{\circ} \mathrm{C}$, so the process kills bacteria but avoid denaturing actin protein. Meat becomes tough and dry when cooked to higher temperatures. ${ }^{12}$

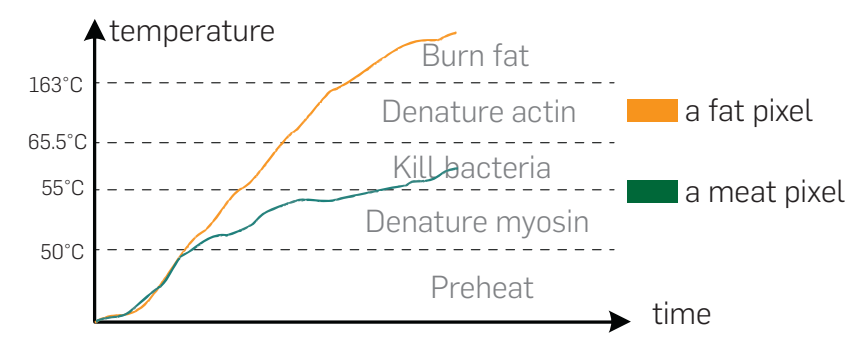

Figure 5. A turntable with 32 neon lights (left) and a plate cover with 32 neon lights (right).

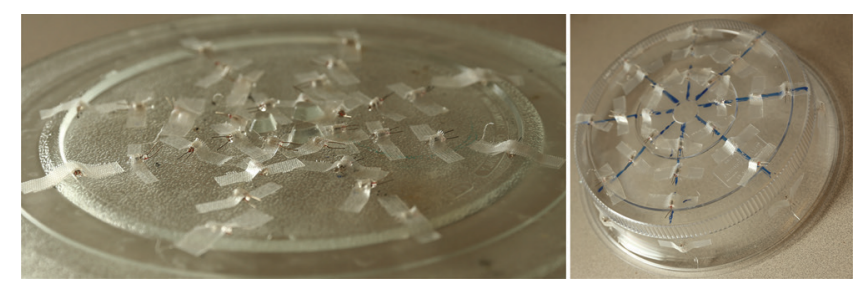


difference, electrons are accelerated away from the cathode and give rise to collisions with the neon gas molecules, which will emit a characteristic glow. The brightness of the lamp is proportional to the EM-field strength at the placed location, and SDC leverages that brightness to measure the EM-field strength.

The glow of the light is sensed by a visible light camera outside the chamber to capture real-time EM-field strength (see Figure 6). However, neon lights are often blocked from direct view of the camera due to obstructions such as food on the turntable. To mitigate this, SDC conducts the light from the neon lamps to the camera using optic fibers (see Figure 7).

Is SDC's hardware microwave safe? Neon lights are microwave-safe because the metal electrodes are encapsulated with a glass capsule, and because the gas glow discharge can avoid energy accumulation. Each neon light consumes minimal microwave energy ( $\approx 19.5 \mathrm{~mW})$, producing negligible interference to existing EM-field patterns. Glass optical fibers are also microwave-safe.

The cameras used in SDC are not affected by the microwave because they are placed outside the microwave oven. The holes created for the optical fibers and the thermal camera are smaller than the $1 / 20$ wavelength of $2.4 \mathrm{GHz}$ radio, so the chamber remains a Faraday cage. The leakage through holes is negligible. Indeed, many commercial microwaves have holes of similar dimensions to support the turntable or stirrer fan.

Programming EM sensitivity: Much like EM fields in radio communication, it is important to tune neon lights to the correct range of sensitivity to accurately sense EM-field

Figure 6. The turntable inside a running oven. The brightness of a neon light is proportional to the EM-field strength at the placed location.

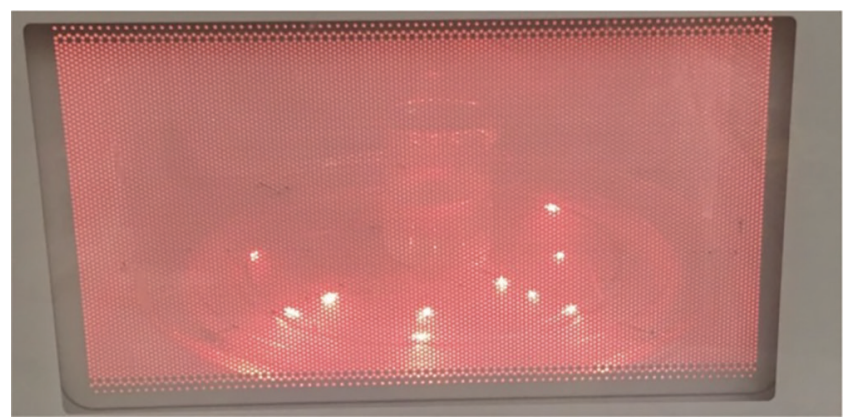

Figure 7. The optical fiber carries the signal outside of the chamber.

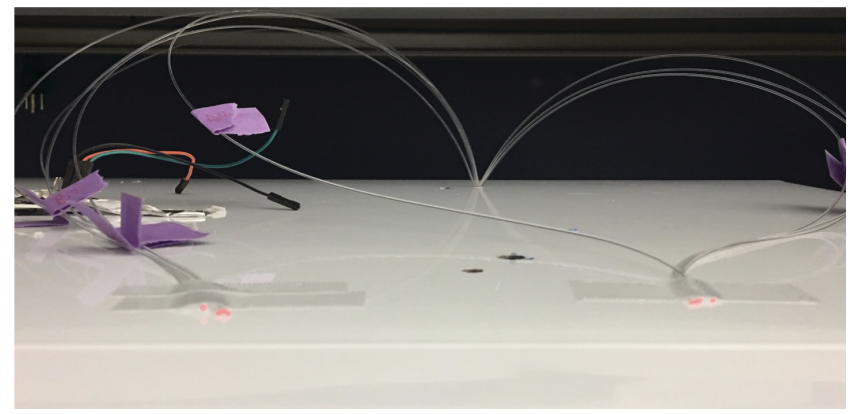

strength. We define sensitivity of the neon light as the change in brightness for a given change in EM-field strength. A highly sensitive neon light may be saturated by a strong EM field and burn the antennas, whereas a poorly sensitive neon lamp may not light up under a low EM field.

To find the right level of sensitivity, we tested neon lights with several different lengths of wire extensions (see Figure 2). Specifically, depending on the EM-field strength, a neon light may experience one of the following three states when running the microwave: Consistently off, flashing at various intervals, and consistently on. The flashing (flashing frequency) and consistently on (brightness) states offer more fine-grained resolution of the EM field than consistently off. An ideal neon light sensitivity would ensure that a good percentage of the neon lights in the oven are in the flashing and consistently on states. To tune the optimal antenna length (i.e., the wire extension length), we empirically tested various types of loads in the microwave and found that a wire extension of $8 \mathrm{~mm}$ achieves the desired sensitivity.

\subsection{Modeling heat over time and space}

Next, we describe how we measure the brightness and flashing frequency of strategically placed neon lamps, and use this data to model the current and future temperature of food over 3D space and time.

Creating a spatial heatmap: The visual camera, placed at the front of the microwave oven, captures the brightness of neon lights in a real-time video stream. SDC measures the brightness of the lamps every 0.1 seconds (i.e., 12 frames of a $120 \mathrm{fps}$ video stream). For each frame, SDC converts the image to grayscale, finds the pixels around the neon light or at the end of an optical fiber, and sums up the pixel values as the brightness score. Because the locations of the neon lights are known a priori, we interpolate the brightness at remaining locations using cubic-spline interpolation. We then map neon light brightness and flashing frequencies to EM-field strength empirically by comparing results from colocated neon lamps. This, coupled with spatial interpolation, allows us to generate a 3D EM-field intensity view within the microwave chamber. SDC can therefore estimate the EM-field strength, given a specific location at finegrained spatial resolution.

Modeling temperature over space-time: As mentioned previously, SDC can measure the current temperature of the food surface by placing a thermal camera on the roof of the microwave oven to sense the food surface in a top-down view. However, using a thermal camera exclusively to model temperature has two limitations: (1) the camera only measures temperatures on the food surface in its direct field-ofview; (2) the camera only measures the current temperature and not future expected temperature.

SDC estimates future heat by integrating measurements of the EM field obtained from the neon lamps. Specifically, the heating of any given point in space of the food is proportional to the EM-field intensity of that location. This means that integrating the observed field intensity, while accounting for the rotation of food over time, can provide a robust estimate of its future temperature. Yet, two challenges remain in making this mapping accurate: (1) first, the temperature of the 
food for the very same EM field may change owing to the material composition of the food itself; (2) second, integrating EM-field intensities over time may cause errors to build up progressively as well.

SDC mitigates the limitations of both the thermal camera system and the EM-field estimation by combining them and obtaining the best of both worlds. Specifically, at each point in time we reconcile the integration of EM-field estimates with those of the thermal camera over space. We then use this to refine our model for mapping EM field to temperature. We repeat this process over time to continuously avoid any drift of our EM field to temperature mapping, as well as accounting for material properties.

\section{SDC'S HEAT ACTUATION}

Today's microwave ovens actuate the heating process in a crude manner. The oven turntable rotates the food blindly without any precise control. The magnetron, the heating engine of the microwave oven, achieves power control by periodically turning itself on and off. SDC augments the existing blind actuation hardware into a closed-loop control system by incorporating the results of heat sensing.

\subsection{Actuation hardware}

Smart turntable: We modify the default turntable inside a commercial microwave oven. More specifically, we replaced the motor with a low-cost stepper motor, and 3D printed a plastic coupler between the motor head and the glass platform to enable precise control. We also connected the magnetron to an Arduino and programmatically sent pulsewidth modulation signals to control the ON/OFF of the magnetron. Rotating the food around can manipulate the heat pattern mildly (e.g., uniform heating), but it is insufficient to create an arbitrary heat pattern.

Programmable accessories: To achieve a more skewed heating pattern, we developed programmable accessories (see Figure 8) that leverage the reflective property of microwave heating, redirecting energy toward desired locations and shielding undesired locations, to achieve an arbitrary heating capability.

To minimize RF energy transfer at specific regions, we installed a horizontal glass plane above the turntable and carefully packed metallic spheres (see Figure 8 right) correspondingly. Metallic spheres by definition do not have edges and are therefore microwave-safe. These metallic spheres

Figure 8. Programmable microwave accessories.

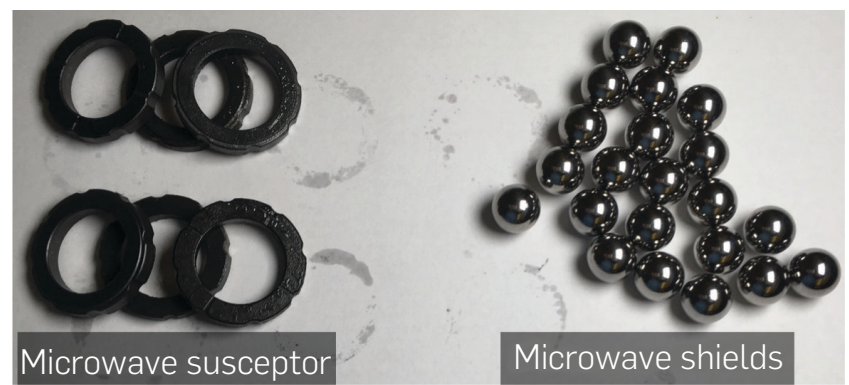

effectively form a microwave mirror to reflect microwave energy at the specific region.

The most common dielectric dipole in the food is water, so microwave heating rarely achieves temperature beyond the boiling point of water. However, some important food chemical reactions occur well above water's boiling point, such as Maillard reactions and caramelization. We introduce microwave susceptors to address this limitation. Materials like silicon carbide (see Figure 8 left) can effectively absorb microwave energy inside the oven and reach $200+{ }^{\circ} \mathrm{C}$ within 1 min microwaving. Attaching silicon carbide to the food surface can then trigger desired high-heat reactions.

\subsection{Recipe and actuation representation}

Having developed programmable actuation hardware, this section formally states the actuation optimization problem that attempts to heat the food in accordance with the input heating recipe.

Heating recipe: An SDC heating recipe will specify the desired temperature trajectory and duration for each part at different temperatures. Mathematically, we can formulate the recipe as follows. Let us imagine that the food surface is divided into a set of discrete pixels. Given $n$ pixels $B=\left\{B_{1}, B_{2}, \ldots B_{n}\right\}$ on the surface of the food, the 3D coordination of the pixels is $\left\{x_{i}, y_{i}, z_{i}\right\}$, where $i \in\{1,2, \ldots n\}$. The recipe is a mapping function $f$ that maps the pixels and the timestamps to desired temperatures throughout the $D$ min cooking journey:

$$
f\left(B_{i}, j\right)=p_{i j}, \quad i \in\{1,2, \ldots n\} \quad 0<j<D
$$

where $j$ denotes the timestamp since start of the cooking process, and $p_{i j}$ refers to the desired temperature for $i$-th pixel at the timestampj.

SDC's optimization problem: Our goal of the smart turntable is to find a rotation plan $S^{*}$ that can move food in and out of these hot and cold spots as needed to cook food according to the desired heat trajectory $P$, which contains collection of desired temperatures $p_{i j}$ across the space and time. SDC defines a rotation plan $S$ as a sequence of angle-duration and magnetron on-off-duration tuples:

$$
\begin{aligned}
S & =\left[\begin{array}{l}
\left\{\theta_{1}: d_{\theta 1}\right\},\left\{\theta_{2}: d_{\theta 2}\right\},\left\{\theta_{3}: d_{\theta 3}\right\}, \ldots \\
\left\{o_{1}: d_{o 1}\right\},\left\{o_{2}: d_{o 2}\right\},\left\{o_{3}: d_{o 3}\right\}, \ldots
\end{array}\right] \\
D & =\sum\left\{d_{\theta 1}, d_{\theta 2}, d_{\theta 3}, \ldots\right\}=\sum\left\{d_{o 1}, d_{o 2}, d_{o 3}, \ldots\right\}
\end{aligned}
$$

where $\left\{\theta_{k}: d_{\theta k}\right\}$ indicates that the turntable will stay at the absolute offset angle $\theta_{k}$ for a duration of deu, $\left\{o_{k}: d_{o k}\right\}$ describes the duration $d_{o k}$ for keeping the magnetron on or off $\left(o_{k}\right)$. Based on these definitions, we now formulate SDC's core optimization problem as follows:

$$
S^{*}=\arg \min _{S} \sum\|\bar{P}(S)-P\|^{2}
$$

where $P(S)$ denotes the temperature trajectory for the $\mathrm{n}$ pixels using a rotation plan $S$ over time. 


\subsection{Actuation algorithm}

Solving this optimization problem is challenging for two reasons. First, microwaves heat the food through a standing wave, so they cannot heat individual pixels independently. Heating one pixel will inevitably heat other pixels as well. To achieve the desired heat pattern, we need to select a set of heat patterns whose union is equivalent to the target heat pattern. Second, the heat pattern, the result of the EM-ingredient coupling, is nonstatic and unpredictable. The EM-field distribution changes gradually when the turntable rotates the food and when the food heats up. SDC cannot predict the output heat pattern until the food is heated.

The stochastic knapsack: At a high level, this problem is a variant of the stochastic knapsack problem, ${ }^{11}$ a classic resource allocation problem of selecting a subset of items to place into a knapsack of given capacity. Placing each item in the knapsack consumes a random amount of the capacity and provides a stochastic reward, ${ }^{11}$ which is only observable after the item is placed. Due to the intrinsic uncertainty of stochastic knapsack problems, adaptive and closed-loop strategies often perform better than open-loop ones in which the items chosen are invariant of the remaining time budget. ${ }^{2}$

Approximation algorithm: We propose a greedy approximation algorithm to determine the immediate rotation plan on-the-fly based on the sensing result in section 4 . Our greedy strategy is as follows: "at each step of the journey, heat at the rotation angle whose temperature gradient is most similar to the current heating gap".

Figure 9 illustrates the workflow of the greedy algorithm. In SDC, the thermal camera continuously senses the current food temperature at the $n$ pixels: $C=\left\{c_{1}, \mathrm{c}_{2}, \ldots, c_{n}\right\}$. SDC then compares the desired heating pattern $f\left(B_{i}, j\right)$ with the observed thermal distribution, and computes the real-time heating gap $G=\left\{g_{1}, g_{2}, \ldots, g_{n}\right\}$. When running, SDC continuously computes the similarity between the temperature gradient at $\theta$ and the current heating gap using the cosine of an angle between these two vectors. Once computed, SDC rotates the turntable to $\theta^{*}$, which has the most well-aligned temperature gradient. Simultaneously, SDC updates the dictionary of temperature gradients based on the EM field and real-time observation of temperature from the IR camera at

Figure 9. Workflow of SDC's heat actuation: (1) SDC first computes the heating gap by comparing the desired heating pattern with the current status from the thermal camera. (2) SDC then adjusts the actuation plan accordingly and (3) updates the distribution continuously.

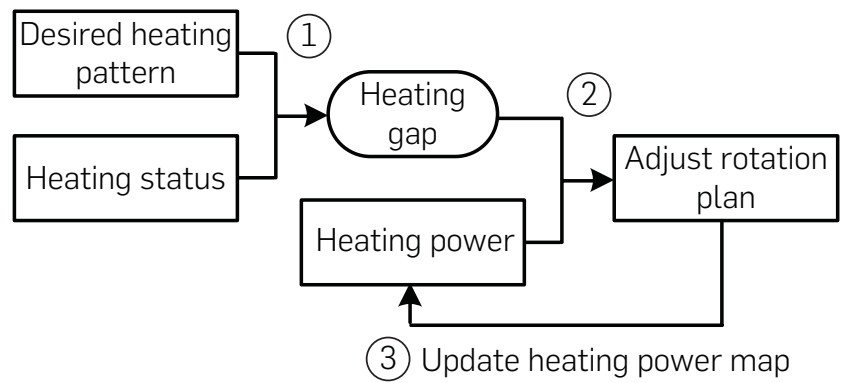

each rotation angle $\theta$.

\section{IMPLEMENTATION AND EVALUATION}

We implement SDC by modifying a commercial Microwave oven (Sharp SM1441CW) and conduct experiments with a variety of food types, including meat and rice, to evaluate SDC's heat sensing and actuation.

Ground truth: To obtain ground-truth temperature data, we use a noncontact IR thermometer (Etekcity Lasergrip 630), which provides $\pm 2^{\circ} \mathrm{C}$ resolution from $-50^{\circ} \mathrm{C}$ to $580^{\circ} \mathrm{C}$, as well as the thermal camera we used in the SDC.

\subsection{Uniform heating}

Nonuniform heating is a major drawback associated with today's microwaves, ${ }^{18}$ which not only affects the quality of the food but also compromises food safety when the microorganisms may not be destroyed in the cold spots. This experiment evaluates SDC when provided with a Uniform heating plan, a common input thermal trajectory provided to SDC, in which we aim to heat all the pixels to the same temperature at a uniform pace.

Method: We conduct our evaluation by heating raw rice grains using SDC. To visualize the heat pattern, we color the grains with thermal-chromatic pigment (see Figure 10), which will turn into pink as the temperature increases. We use the thermal-chromatic pigment approach because it can provide a rich and analog temperature visualization, while thermal cameras have a limited resolution and the final output images are based on interpolation.

We begin our experiment at a room temperature of $20^{\circ} \mathrm{C}$. We create a uniform heating recipe that requires that the food be heated uniformly from $20^{\circ} \mathrm{C}$ to $60^{\circ} \mathrm{C}$ over 2 min over space as per the recipe provided by the thick blue line in Figure 11. We note that the thermal trajectory is identical across all pixels of the food surface. However, the temperature increase is not designed to be linear over time, instead mimicking the smoothed average temperature trajectory for raw rice within a microwave under normal microwave operation.

To characterize the benefits of SDC, we use two cases of blind microwave heating as the baselines: The same microwave oven (1) with and (2) without turntable rotation. To collect the immediate temperature during heating, we take out the food every $30 \mathrm{sec}$ to measure ground truth.

Figure 10. We color rice grains with thermal-chromatic pigment, which turn pink in a predictable manner as their temperature increases.

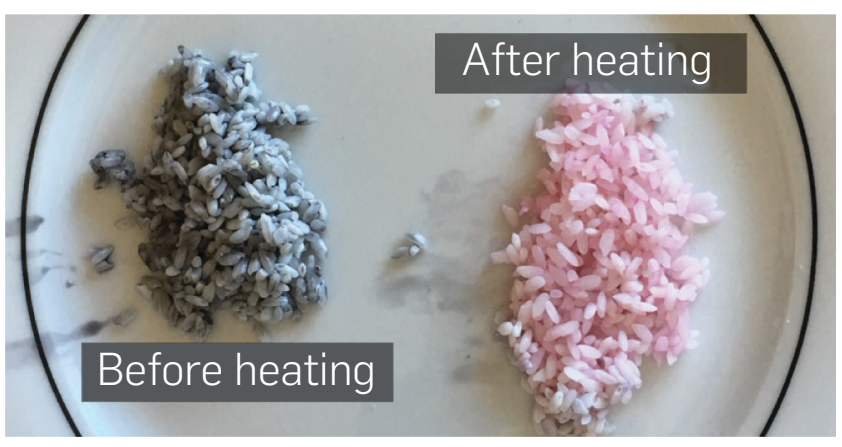


Results: Figure 12 shows a visualization of the thermalchromatic pigment, which changes color at $31^{\circ} \mathrm{C}$ and progresses to darker shades of pink with increased temperature. The rice colored (dull white) regions denote spots of food that remain below $31^{\circ} \mathrm{C}$. We observe that SDC achieves a uniform pink hue that darkens over time, whereas the baselines (no rotation or default rotation) continue to have cold spots through time. Note that SDC visually appears to have the deepest shade of pink vs. the baselines at $\mathrm{t}=120 \mathrm{sec}$ as it achieves more spatially uniform temperature relative to the baselines. In actuality, there are also some hot spots of the baseline schemes that achieve even higher temperatures (over $70^{\circ} \mathrm{C}$ ), whereas SDC

Figure 11. (above) Temperature variance of SDC uniform heating is the lowest; (below) points on food closely follow heating recipe.

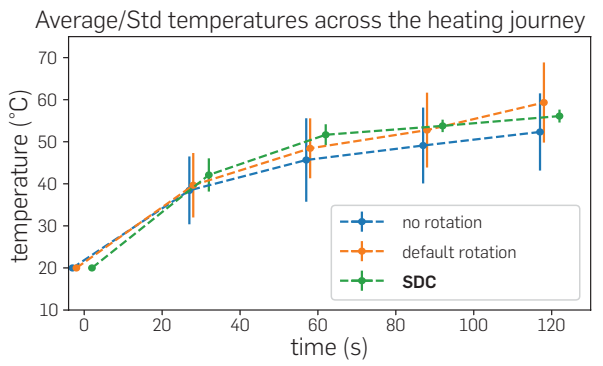

Thermal trajector for 9 discrete points and the input recipe

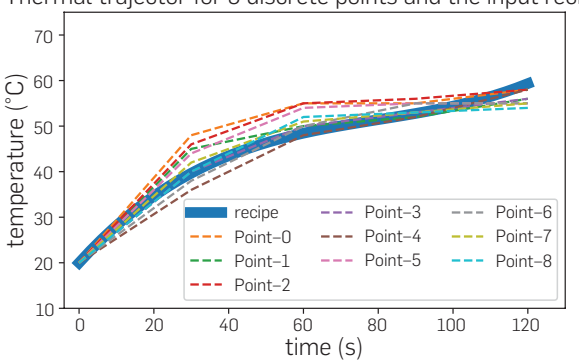

achieves uniform temperature closer to $60^{\circ} \mathrm{C}$ as desired.

Validating heat sensing and actuation: Figure 11 (bottom) shows the trajectory of the temperature over time for nine discrete uniformly spaced points of the food using SDC. Note that all points closely follow the recipe over time, which demonstrates SDC's high accuracy in modeling the temperature gradient. Figure 11 (top) compares the average and standard deviation of the trajectory across the same discrete points measured over multiple experiments vs. time. Of particular interest here is the standard deviation of the temperature of the food where one can clearly observe that SDC achieves a lower spatial variance in temperature when compared to the baseline schemes. This validates our findings that although microwaves heat food blindly and nonuniformly, SDC can achieve significant uniformity in heating.

Our results validate the correctness of both heat sensing and actuation, both of which must operate correctly to achieve the desired heating objective.

\subsection{Arbitrary heating}

In real-world cooking, different ingredients often require to be cooked at different temperatures. SDC can support these activities computationally by specifying thermal trajectories for different surface pixels. In this section, we aim to stress test SDC by exploring the maximum heating resolution, that is, the maximum temperature difference that can be created in a fine-grained spatial resolution.

Method: We create an imaginary recipe that heats a unique thermal pattern (depicted in Figure 13 left). The recipe sets the target temperature for the inner ring area at $500^{\circ} \mathrm{C}$ and the rest area at $50^{\circ} \mathrm{C}$. We deliberately set an unachievable goal of $500^{\circ} \mathrm{C}$ for SDC to stress test the system and evaluate how well SDC can approximate to the targets. We conduct two independent experiments with and without the help of microwave accessories. To characterize the performance, we focus on the peak-to-peak temperature differences $\Delta P$ (i.e., the difference

Figure 12. Visualization of heating of rice as a function of time for no rotation, default rotation, and SDC. The dull white regions denote spots of undercooked rice. SDC results in the most uniform heating.

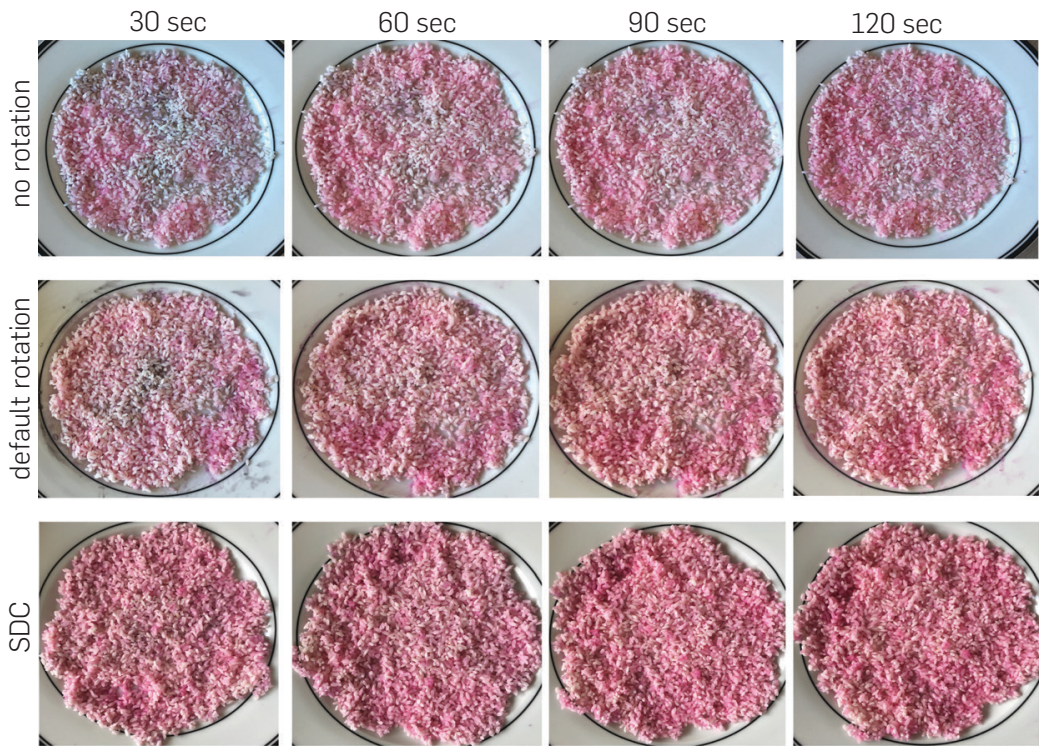


Figure 13. Left: An input recipe for stress test. Middle: SDC without susceptors. Right: SDC with susceptors. Susceptors can help build more skewed thermal distributions.

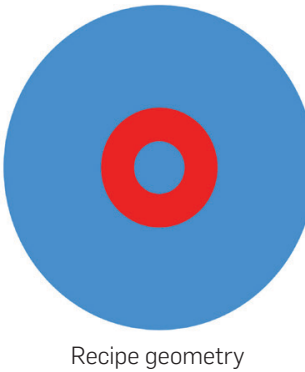

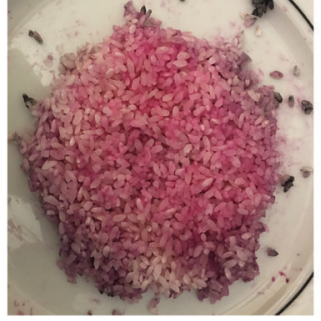

SDC without accessories

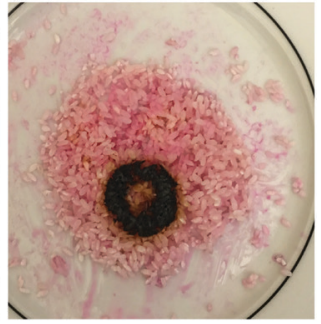

SDC with accessories between the maximum and the minimum temperature).

Results: Figure 13 (right) shows a visualization of the thermal-chromatic pigment, which aligns well with the desired pattern (Figure 13 left), without and with microwave accessories. As expected, we observe that the presence of accessories helps improve the contrast between the high temperature and low temperature rings. This is precisely why accessories are needed to improve SDC's performance during tasks such as searing, where extreme temperature gradients are needed on the food.

Figure 14 on the right summarizes the mean $\Delta P$ and standard deviation $\sigma_{p}$ of the maximum temperature difference between the inner and outer rings achieved in SDC with and without microwave accessories. We also note that with microwave accessories, SDC can cause extremely high temperature gradients (up to $61^{\circ} \mathrm{C}$ per centimeter) at very fine spatial resolution.

\subsection{App-Cooking meat (Bacon)}

In this section, we evaluate SDC's performance in cooking with advanced thermal recipes. We consider bacon with different heating requirements for the meat and fat.

Method: We use the heating recipe required per pixel for the meat vs. the fat as shown in Figure 4. Based on the package instruction, we set the heating time to $1 \mathrm{~min}$. We place strips of bacon on the microwave plate across multiple experiments. We measure the continuous ground-truth temperature using the thermal camera and the final temperatures at discrete points using the noncontact IR thermometer (Etekcity Lasergrip).

Results: Figure 15 depicts the initial and final product over the cooking process. Note that although default rotation heats the bacon unevenly (resulting in the uneven shape), SDC heats the microwave more uniformly while differentiating between the heat applied to meat and fat. Indeed, we observe that the cooking process does not overcook/burn the meat, while at the same time avoiding colder spots that may pose a health hazard.

\section{RELATED WORK}

Related work falls under three broad categories:

Sensing in microwaves: There has been much past related work on improving the heat sensing within microwave ovens. For example, advanced FISO Microwave Work Stations (MWS) ${ }^{3}$ used in food research have special microwave-safe fiber-optic
Figure 14. Mean $(\Delta P)$ and standard deviation $\left(\sigma_{\rho}\right)$ of thermal delta for arbitrary heating. The final column $\frac{\Delta P}{d}$ denotes the temperature gradient per unit distance that can be ăchieved.

\begin{tabular}{|l|c|c|c|}
\hline Scheme & $\Delta P$ & $\sigma_{P}$ & $\frac{\Delta P}{d}$ \\
\hline without accessories & $21^{\circ} \mathrm{C}$ & $99^{\circ} \mathrm{C}$ & $3^{\circ} \mathrm{C} / \mathrm{cm}$ \\
\hline with accessories & $183^{\circ} \mathrm{C}$ & $42^{\circ} \mathrm{C}$ & $61^{\circ} \mathrm{C} / \mathrm{cm}$ \\
\hline
\end{tabular}

Figure 15. The raw bacon and slices of bacon cooked with SDC and the original turntable. These three slices of bacon are from the same package, so the original fat patterns are nearly identical. Heated meat and fat will shrink. SDC applies heat to meat and fat discriminatively, so the fat shrinks more than the meat.

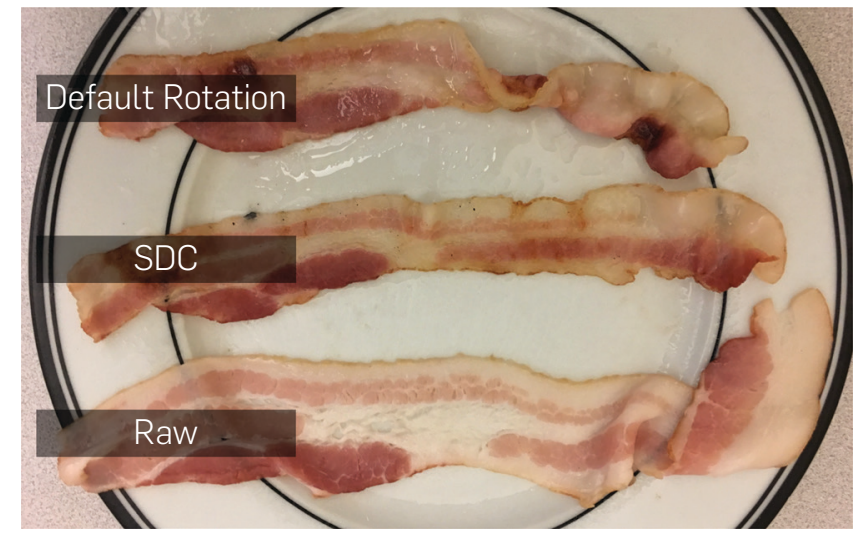

sensors to collect real-time fine-grained direct measurements inside the cavity, but cost $\$ 80 \mathrm{k}+$. Researchers ${ }^{8}$ have also used software radios to monitor the signal strength of the microwave leakage and recognize the type of food. However, many variables, such as food type, quantity, temperature, and food location inside the oven impact microwave leakage unpredictably. ${ }^{19}$ In contrast to these systems, SDC estimates both current and future temperature distributions by directly placing low-cost microwave-safe sensors within the cavity and modeling EM propagation.

Actuation in microwaves: The most widely adopted microwave actuation is the turntable and the stirrer blade ${ }^{22}$ that attempt to spread radiation uniformly. However, these blind actuation approaches cannot eliminate hot/cold spots, due to the inherent unpredictability of the EM-field 
distribution. More recent advances in the microwave generators, such as an RF solid-state cooker, adjust the transmitter's real-time power, frequency, and phase to move the hot/cold spots around, albeit at high cost $\left(\sim \$ 10,000^{9}\right)$ and complexity. SDC also draws inspiration from many microwave accessories that have been developed to cook certain foods in a microwave-for example, Corning Ware Microwave Browners, Microwave egg boilers, or the susceptors in popcorn bags. Unlike this past work, SDC provides a generalized framework for heat actuation as per a userspecified thermal trajectory as well as the sensing results, without being tied to specific types of food or adding expensive components.

Computational fabrication and heating: Designing computational fabrication techniques ${ }^{15}$ for digital gastronomy is an emerging topic. ${ }^{23}$ The most relevant approach is laser cooking, ${ }^{5}$ which uses a computer-controlled laser cutter to heat a sequence of small spots of the food surface. While innovative, the rolling pixel-by-pixel heating process is known to be highly time-consuming. SDC overcomes the slow production time of laser heating while allowing for a high degree of flexibility in the numerous heating patterns produced.

\section{CONCLUSION}

In this work, we presented Software-Defined Cooking (SDC), a novel next-generation microwave oven that both senses and actuates heating at fine-grained spatial resolution. Among all three common heating methods (convection, conduction, and radiation), radiation is the only one that can redirect energy toward the desired location. This redirectable feature makes the microwave oven an ideal platform to experiment with SDC, as it can effectively program energy transferring without physical hardware changes.

Meanwhile, although there has been a great deal of past work on novel RF applications for communication, ${ }^{14}$ sensing; ${ }^{7}$ and energy harvesting, ${ }^{20}$ novel actuation mechanisms using RF signals are less explored so far. SDC is designed to innovate in this space.

Our current prototype already demonstrates a promising result in controlled RF actuation. With more sophisticated power transfer mechanism development (e.g., inexpensive ways of performing high-power RF beamforming), we think that future well-engineered RF cooking devices can be the ultimate cooking equipment for every family, achieving cooking quality and convenience at the same time.

\footnotetext{
References

1. Ackerman, E. A brief history of the microwave oven - IEEE spectrum. https://spectrum.ieee.org/techhistory/space-age/a-brief-historyof-the-microwave-oven, 2016. (Accessed on 02/10/2019).

2. Dean, B.C., Goemans, M.X., Vondrák, J. Approximating the stochastic knapsack problem: The benefit of adaptivity. Math. Oper. Res. 33, 4 (2008), 945-964

3. FISO Technologies Inc. Mws microwave work station - product datasheet, 2018. http://tech-quality. com/images/stories/pdf/OT/mws.pdf (Accessed on 11/21/2018).

4. Food Safety and Inspection Service, United States Department of Agriculture. Appendix a to
}

compliance guidelines, 1999 https://www.fsis.usda.gov/Oa/ fr/95033f-a.htm?redirecthttp=true (Accessed on 02/21/2019). Takao, S. Laser cooking: A novel culinary technique for dry heating using a laser cutter and vision technology. In Proceedings of the ACM Multimedia 2012 Workshop on Multimedia for Cooking and Eating Activities (2012), ACM, New York, NY, USA, 55-58.

6. Howell, E. Things you shouldn't cook in a microwave I microwave safety, 2013. https://www.livescience. com/34406-microwave-safetyexperiments.html. (Accessed on 03/17/2019)

7. Jin, H., Yang, Z., Kumar, S., Hong, J.I.
5. Fukuchi, K., Jo, K., Tomiyama, A.,
Towards wearable everyday body-frame tracking using passive rfids. Proc. ACM Interact.

Mobile Wearable Ubiquitous Tech. 1, 4 (2018), 145.

8. Kawahara, Y., Bian, X., Shigeta, R., Vyas, R., Tentzeris, M.M., Asami, T. Power harvesting from microwave oven electromagnetic leakage. In Proceedings of the 2013 ACM International Joint Conference on Pervasive and Ubiquitous Computing UbiComp'13 (2013), ACM, New York NY, USA, 373-382.

9. Manz, B. Rf energy is finally cooking, 2017. https://www.mwrf.com/ community/rf-energy-finally-cooking (Accessed on 03/15/2019)

10. Osepchuk, J.M. A history of microwave heating applications. IEEE Trans. Microwave Theory Tech 32, 9 (1984), 1200-1224.

11. Pike-Burke, C., Grunewalder, S. Optimistic planning for the stochastic knapsack problem. In Proceedings of the $20^{\text {th }}$ International Conference on Artificial Intelligence and Statistics. A. Singh and J. Zhu, eds. Volume 54, Proceedings of Machine Learning Research. PMLR, Fort Lauderdale, FL, USA, 2017, 1114-1122.

12. Potter, J.. Cooking for Geeks: Real Science, Great Hacks, and Good Food. O'Reilly, 2016.

13. Sloan, A.E. Demographic redirection. Food Tech. 67, 7 (2013), 38-49

14. Talla, V., Kellogg, B., Gollakota, S. Smith, J.R. Battery-free cellphone. Proc. ACM Interact. Mobile Wearable Ubiquitous Tech. 1, 2 (2017), 25

15. Torres, C., Chang, J., Patel, A., Paulos, E. Phosphenes: Crafting resistive heaters within thermoreactive composites. In Proceedings of the 2019 on Designing Interactive Systems Conference, DIS'19

Haojian Jin and Jason Hong (\{haojian@ cs., jasonh@cs.]cmu.edu), HumanComputer Interaction Institute, School of Computer Science, Carnegie Mellon University, Pittsburgh, PA, USA
(2019), ACM, New York, NY, USA, 907-919.

16. United States Department of Agriculture. Microwave ovens and food safety, 2013. https://www.fsis usda.gov/wps/portal/fsis/ topics/food-safety-education get-answers/food-safety-fact-sheets/ appliances-and-thermometers/ microwave-ovens-and-food-safety/ ct_index. (Accessed on 02/14/2019).

17. U.S. Food \& Drug Administration. Microwave oven radiation safety standard, 2017. https://www.fda. gov/radiation-emittingproducts/ resourcesforyouradiationemittingproducts/ ucm252762.htm. (Accessed on 02/10/2019)

18. Vadivambal, R., Jayas, D. Non-uniform temperature distribution during microwave heating of food materials - A review. Food Bioprocess Tech. 3, 2 (2010), 161-171.

19. Vollmer, M. Physics of the microwave oven. Phys. Educ. 39, 1 (2004), 74.

20. Wang, J., Zhang, J., Saha, R. Jin, H., Kumar, S. Pushing the range limits of commercial passive rfids. In $16^{\text {th }}$ USENIX Symposium on Networked Systems Design and Implementation (NSDI 19) (2019) USENIX Association, Boston, MA, 301-316.

21. Wikipedia. Dielectric heating Wikipedia, 2018. https://en.wikipedia. org/wiki/Dielectric_heating. (Accessed on 09/05/2018).

22. Yu, H.-S. Microwave Oven with a Turntable and Mode Stirrers, US Patent 5,877,479, 21999.

23. Zoran, A., Cohen, D. Digital konditorei: Programmable taste structures using a modular mold. In Proceedings of the 2018 CHI Conference on Human Factors in Computing Systems (2018), ACM, New York, NY, USA, 400.
Jingxian Wang and Swarun Kuma (\{jingxian@, swarun@\}cmu.edu), Department of Electrical and Computer Engineering, Carnegie Mellon University, Pittsburgh, PA, USA. 